\title{
QUASI-HEREDITARY ALGEBRAS
}

\author{
BY \\ Vlastimil Dlab and Claus Michael Ringel
}

\section{Dedicated to the memory of Irving Reiner}

In their work on highest weight categories arising in the representation theory of Lie algebras and algebraic groups, E. Cline, B. Parshall and L. Scott recently introduced the notion of a quasi-hereditary algebra (see [1] and [2]). They define a quasi-hereditary algebra recursively in terms of the existence of a particular idempotent ideal; finite-dimensional hereditary algebras are typical examples of quasi-hereditary algebras. On the other hand, they showed that every quasi-hereditary algebra has finite global dimension.

The purpose of this note is to establish the following three results. First, finite-dimensional hereditary algebras are characterized as those quasi-hereditary algebras which satisfy a certain refinement property on chains of their idempotent ideals (Theorem 1). Second, all finite-dimensional algebras of global dimension 2 are shown to be quasi-hereditary (Theorem 2). Third, the question of whether every finite-dimensional algebra of finite global dimension is quasi-hereditary is answered in the negative by providing an example of an (11-dimensional serial) algebra of global dimension 4 which is not quasihereditary. The same example illustrates that the class of quasi-hereditary algebras is not closed under tilting (in the sense of [4]).

In what follows, all rings are semiprimary rings. An associative ring $A$ with 1 is called semiprimary if its Jacobson radical $N$ is nilpotent and $A / N$ is semisimple artinian. Recall that an ideal $I$ of $A$ is idempotent if and only if $I=A e A$ for an idempotent $e$ of $A$; in particular, $I$ is a minimal (non-zero) idempotent ideal provided that $e$ is primitive. An ideal $J$ of $A$ is said to be a heredity ideal of $A$ if $J^{2}=J, J N J=0$ and $J$, considered as a right $A$-module $J_{A}$, is projective. In fact, this also implies that the left $A$-module ${ }_{A} J$ is projective (see [2] or [3]). A semiprimary ring $A$ is called quasi-hereditary if there is a chain

$$
0=J_{0} \subset J_{1} \subset \cdots \subset J_{t-1} \subset J_{t} \subset \cdots \subset J_{m}=A
$$

of ideals of $A$ such that, for any $1 \leq t \leq m, J_{t} / J_{t-1}$ is a heredity ideal of $A / J_{t-1}$. Such a chain of idempotent ideals is called a heredity chain. Let us

Received December 12, 1987. 
remark that $A$ is quasi-hereditary if and only if $A^{\text {op }}$ is quasi-hereditary (see [2] or [3]).

THEOREM 1. Let $A$ be a semiprimary ring. Then $A$ is hereditary if and only if every chain of idempotent ideals of $A$ can be refined to a heredity chain.

Proof. First, let $A$ be a hereditary semiprimary ring. Note that, for any idempotent ideal, the ring $A / I$ is again hereditary. Thus, it is sufficient to show that any non-zero idempotent ideal $I$ of $A$ contains a non-zero heredity ideal. To this end, consider $J=A e A$ with a primitive idempotent $e$ in $I$. Since $A$ is hereditary, $J_{A}$ is projective. Moreover, since the left multiplication by any element $x \in e N e$ defines a non-invertible map from the indecomposable module $e A$ onto $x A$ and $x A$ is projective, necessarily $x=0$. It follows that $J N J=A e N e A=0$.

Conversely, assume that every chain of idempotent ideals of $A$ can be refined to a heredity chain. We want to show that $A$ is hereditary. We shall proceed by induction; thus assume that $A / I$ is hereditary for every non-zero idempotent ideal $I$ of $A$.

Let us start by choosing a primitive idempotent $f$ in $A$ such that the Loewy length $L(f A)$ of the module $f A$ is maximal. Observe that $I=A f A$ is a heredity ideal. Indeed, $A f A$ is a minimal idempotent ideal of $A$. Thus $f N f=0$ and $I_{A}$ is a projective $A$-module. We claim that $N f=0$. For, if $N f \neq 0$ then there is a primitive idempotent $e$ such that $e N f \neq 0$ and thus $e N f=e A f$. Furthermore, $I=e I \oplus(1-e) I$, and thus $(e I)_{A}$ is a projective $A$-module. Since $e I=e A f A$ is generated by $f A$, it is a non-zero direct sum of copies of $f A$ and therefore $L(e I)=L(f A)$. On the other hand, $e I=e A f A=e N f A \subseteq e N$, and consequently

$$
L(e I) \leq(e N)=L(e A)-1
$$

which contradicts the maximality of $L(f A)$. Hence, $N f=0$.

Now, write $1=\sum_{i=1}^{n} e_{i}$ with primitive orthogonal idempotents $e_{i}$. Assume that $f=e_{1}$ is equivalent to $e_{i}$ if and only if $1 \leq i \leq k$. Thus $e_{j} A e_{i}=0$ for all $i \leq k<j$. If $k=n$, then $A$ is simple artinian and thus hereditary. If $k<n$, we show that $(A f N)_{A}$ is a projective $A$-module. For then, in conjunction with the fact that $N f=0$ and that $B=A / A f A$ is hereditary, we can conclude that $A$ is hereditary, as required.

In order to prove that $(A f N)_{A}$ is projective, consider the $A$-module $X_{A}=$ $A f N /(A f N \cap A g A)$, where $g$ is a primitive idempotent satisfying $N g \subseteq I$ $(=A f A)$. Such an idempotent $g=e_{j}$ for some $j>k$ exists, since $B=A / I$ is 
hereditary. Clearly, $X$ is isomorphic to the ideal

$$
(A f N+A g A) / A g A
$$

of the ring $C=A / A g A$ which is, by induction, hereditary. Consequently, $X$ is a projective $C$-module. Since $X$ is annihilated by $A(f+g) A$, it is a projective $D$-module for $D=A / A(f+g) A$. But, in view of $N g \subseteq I$, every projective $D$-module is a projective $B$-module, and in view of $N f=0$, every projective $B$-module is a projective $A$-module. Hence, $X_{A}$ is a projective $A$-module. As a result, the canonical $A$-homomorphism $(A f N)_{A} \rightarrow X_{A}$ splits and thus $(A f N)_{A}$ is isomorphic to the direct sum of the projective $A$-module $X_{A}$ and $(A f N \cap A g A)_{A}$. However, the latter $A$-module is also projective. This follows from the fact that

$$
A f N \cap A g A=\bigoplus_{i=1}^{k} e_{i} A g A,
$$

which is trivially a direct summand of the projective $A$-module

$$
A g A=\bigoplus_{i=1}^{n} e_{i} A g A
$$

In order to verify $(*)$, notice that, on the one hand,

$$
e_{i} A g A \subseteq A g A \text { and } e_{i} A g A \subseteq\left(A e_{i} A\right)(A g A)=A f N g A \subseteq A f N,
$$

and that, on the other hand,

$$
A f N \cap A g A \subseteq\left(\bigoplus_{i=1}^{k} e_{i} A\right) \cap A g A=\bigoplus_{i=1}^{k} e_{i} A g A .
$$

This concludes the proof of Theorem 1 .

THEOREM 2. A semiprimary ring of global dimension 2 is quasi-hereditary.

The proof is based on the following proposition.

Proposition. Let A be a semiprimary ring of global dimension at most 2 . Let $e$ be a primitive idempotent of $A$ such that the Loewy length $L(e A)$ is minimal. Then AeA is a heredity ideal.

Proof. Write $J=A e A$. Clearly $J^{2}=J$. First, we are going to show that $e N e=0$; this implies immediately that $J N J=0$. Assume that there is a non-zero element $x \in e N e$. The left multiplication by $x$ yields an endomor- 
phism of $e A$ with a non-zero kernel $K \subseteq e N$. Since $\operatorname{gl} \operatorname{dim} A=2$, kernels of maps between projective modules are projective. Thus $K$ is projective and therefore the Loewy length $L(K) \geq L(e A)$. But

$$
L(K) \leq L(e N)=L(e A)-1,
$$

in contradiction to the preceding inequality.

Second, in order to show that $J_{A}$ is projective, consider the minimal projective cover $p: P \rightarrow J_{A}$. We claim that $p$ is an isomorphism. Otherwise, there exists a finitely generated direct summand $P^{\prime}$ of $P$ such that the restriction of $p$ to $P^{\prime}$ is not a monomorphism. Now, $P$ and therefore $P^{\prime}$ is a direct sum of copies of $e A$. We may assume that $P^{\prime}=P^{\prime \prime} \oplus \bar{P}$ with $\bar{P} \simeq e A$ and such that the restriction of $p$ to $P^{\prime \prime}$ is a monomorphism. Denoting by $X$ the image of $P^{\prime \prime}$ under $p$, consider the commutative diagram of $A$-modules

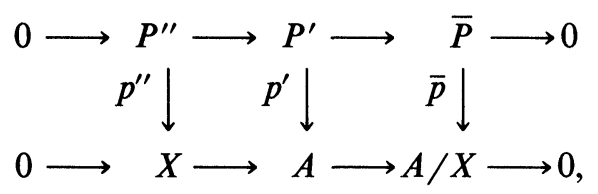

where the horizontal maps are the canonical inclusions and projections, and the vertical ones are induced by $p$ and the embedding of $J$ in $A$. Observe that $p^{\prime}$ is not a monomorphism and $\bar{p} \neq 0$. Forming the kernels, we obtain the exact sequence

$$
0=\operatorname{Ker} p^{\prime \prime} \rightarrow \operatorname{Ker} p^{\prime} \rightarrow \operatorname{Ker} \bar{p} .
$$

Now, since $p^{\prime}$ is a map between projective modules, Ker $p^{\prime}$ is a non-zero projective module, and thus its Loewy length $L\left(\operatorname{Ker} p^{\prime}\right) \geq L(e A)$. But this contradicts the inequality $L\left(\operatorname{Ker} p^{\prime}\right) \leq L(\operatorname{Ker} \bar{p})<L(e A)$. The proof of the proposition is completed.

Proof of Theorem 2. Choose a primitive idempotent $e$ of $A$ such that the Loewy length $L(e A)$ is minimal. Since, by the proposition, $J=A e A$ is a heredity ideal, it follows that $\operatorname{gl} \operatorname{dim} A / J \leq \mathrm{gl} \operatorname{dim} A$ (see [2] or [3]). Therefore, by induction, $A / J$ is quasi-hereditary, and thus $A$ is quasi-hereditary.

Example. Let $A$ be the path algebra (over a field) of the diagram

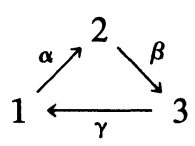

modulo the ideal generated by $\gamma \alpha \beta$ and $\alpha \beta \gamma \alpha$. Denote by $e_{i}$ the idempotent corresponding to the vertex $i$, and let $P_{i}=e_{i} A$. Thus, $A$ is a serial algebra, 
and the lengths of the indecomposable projective $A$-modules are as follows: $P_{1}$ and $P_{2}$ are of length 4 and $P_{3}$ is of length 3 . One can check easily that $\operatorname{gl} \operatorname{dim} A=4$ and that $A$ has no (non-zero) heredity ideals; for, $e_{1} \operatorname{Rad} A e_{1}$ $\neq 0, e_{2} \operatorname{Rad} A e_{2} \neq 0$ and $\left(A e_{3} A\right)_{A}$ is not projective.

Let $S_{2}=P_{2} / \operatorname{Rad} P_{2}$ be the simple $A$-module of projective dimension 1 . Since the modules $P_{1}$ and $P_{2}$ are both projective and injective, it follows that $T=P_{1} \oplus P_{2} \oplus S_{2}$ is a tilting module [4]. Let $B=$ End $T_{A}$. Then $B$ is a quasi-hereditary algebra. Indeed, if $J_{1}$ is the set of all endomorphisms of $T$ which factor through $S_{2}$ and $J_{2}$ the set of all those which factor through $S_{2} \oplus P_{2}$, then $0 \subset J_{1} \subset J_{2} \subset A$ is a heredity chain. This may be verified without difficulty. In fact, one can see easily that $B$ is the path algebra (over a field) of the diagram

$$
1 \underset{\delta}{\stackrel{\alpha}{\rightleftarrows}} 2 \underset{\gamma}{\stackrel{\beta}{\rightleftarrows}} 3
$$

modulo the ideal generated by $\alpha \beta, \alpha \delta, \gamma \delta$ and $\beta \gamma-\delta \alpha$.

\section{Appendix: Heredity ideals}

For the convenience of the reader, we wish to collect here various results of [1] and [2]. In doing so, we aim to minimize assumptions and, at the same time, to strengthen conclusions (cf. examples at the end). Furthermore, we include some assertions from the general ring theory, and provide proofs which are direct and elementary.

In what follows, unless specified otherwise, $A$ is an arbitrary associative ring with $1, J$ and ideal and $B=A / J$. The $B$-modules will always be viewed as those $A$-modules which are annihilated by $J$.

Let us remark that, under the assumption that $A$ is semiprimary, every $A$-module $X$ has finite Loewy length which will always be denoted by $L(X)$. Note that for semiprimary rings, $L\left(A_{A}\right)$ and $L\left({ }_{A} A\right)$ are equal and will be simply denoted by $L(A)$. Always, $L(X) \leq L(A)$.

Statement 1. Let $J_{A}$ be projective. If $X$ is a $B$-module, then

$$
\text { proj } \operatorname{dim} X_{A} \leq 1+\operatorname{proj} \operatorname{dim} X_{B} \text {. }
$$

In particular, proj dim $B_{A} \leq 1$.

Proof. Proceed by induction. The exact sequence $0 \rightarrow J_{A} \rightarrow A_{A} \rightarrow B_{A} \rightarrow 0$ shows that

$$
\operatorname{proj} \operatorname{dim} B_{A} \leq 1 \text {. }
$$


Therefore, for any projective $B$-module $X_{B}, \operatorname{proj} \operatorname{dim} X_{A} \leq 1$. Now, assume that

$$
\text { proj } \operatorname{dim} X_{B}=d>0 \text {, }
$$

and consider an exact sequence $0 \rightarrow X_{B}^{\prime} \rightarrow P_{B} \rightarrow X_{B} \rightarrow 0$ with a projective $B$-module $P_{B}$. Thus proj $\operatorname{dim} X_{B}^{\prime}=d-1$. An application of $\operatorname{Hom}_{A}\left(-, Y_{A}\right)$ yields the exact sequence

$$
\operatorname{Ext}_{A}^{d+1}\left(X_{A}^{\prime}, Y_{A}\right) \rightarrow \operatorname{Ext}_{A}^{d+2}\left(X_{A}, Y_{A}\right) \rightarrow \operatorname{Ext}_{A}^{d+2}\left(P_{A}, Y_{A}\right) .
$$

Since $d \geq 1$ and proj $\operatorname{dim} P_{A} \leq 1$, the last term is zero. By induction,

$$
\text { proj } \operatorname{dim} X_{A}^{\prime} \leq d,
$$

and consequently the first term is zero also. This yields

$$
\operatorname{Ext}_{A}^{d+2}\left(X_{A}, Y_{A}\right)=0
$$

for any $Y_{A}$, and thus proj $\operatorname{dim} X_{A} \leq d+1$.

StATEMENT 2. $J^{2}=J$ if and only if $\operatorname{Hom}_{A}\left(J_{A}, X_{A}\right)=0$ for any $B$-module $X$. If $J$ is projective, then $J^{2}=J$ if and only if $\operatorname{Hom}_{A}\left(J_{A}, B_{A}\right)=0$.

Proof. First, assume that $J^{2}=J$ and let $\phi: J_{A} \rightarrow X_{A}$ be a homomorphism. Then $\phi(J)=\phi\left(J^{2}\right) \subseteq X J=0$, and thus $\phi=0$. Conversely, let $\operatorname{Hom}_{A}\left(J_{A}, X_{A}\right)=0$ for any $B$-module $X$. Write $Y_{A}=J / J^{2}$. Since $Y J=0, Y$ can be viewed as a $B$-module. Hence, $\operatorname{Hom}_{A}\left(J_{A}, Y_{A}\right)=0$, and the canonical epimorphism $J_{A} \rightarrow Y_{A}$ shows that $Y=0$.

Finally, assume that $J_{A}$ is projective and that $\operatorname{Hom}_{A}\left(J_{A}, B_{A}\right)=0$. Given a $B$-module $X$, let $F_{B}$ be a free $B$-module with an epimorphism $\pi: F_{B} \rightarrow X_{B}$. Since $J_{A}$ is projective, any map $\phi: J_{A} \rightarrow X_{A}$ lifts to a map $\phi^{\prime}: J_{A} \rightarrow F_{A}$ with $\phi=\pi \phi^{\prime}$. But $\operatorname{Hom}_{A}\left(J_{A}, F_{A}\right)=0$, because $F_{A}$ is a direct sum of copies of $B_{A}$.

STATEMENT 3. Let $J^{2}=J$ and $J_{A}$ be projective. If $X, Y$ are B-modules, then

$$
\operatorname{Ext}_{B}^{i}\left(X_{B}, Y_{B}\right) \simeq \operatorname{Ext}_{A}^{i}\left(X_{A}, Y_{A}\right) \quad \text { for all } i \geq 0 \text {. }
$$

In particular, $\operatorname{Ext}_{A}^{i}\left(B_{A}, B_{A}\right)=0$ for $i \geq 1$.

Proof. Clearly,

$$
\operatorname{Hom}_{B}\left(X_{B}, Y_{B}\right)=\operatorname{Hom}_{A}\left(X_{A}, Y_{A}\right) .
$$


To prove the case $i=1$, regard $\operatorname{Ext}_{B}^{1}\left(X_{B}, Y_{B}\right)$ as a subgroup of $\operatorname{Ext}_{A}^{1}\left(X_{A}, Y_{A}\right)$. Then, given an exact sequence

$$
0 \rightarrow Y_{A} \stackrel{\mu}{\rightarrow} Z_{A} \rightarrow X_{A} \rightarrow 0
$$

of $A$-modules with an inclusion map $\mu$, it follows, in view of $X J=0$, that $Z J \subseteq \mu(Y)$. Furthermore,

$$
Z J=Z J^{2} \subseteq \mu(Y) J=0,
$$

and thus $Z$ is a $B$-module. This completes the proof for $i=1$.

Finally, let $0 \rightarrow X_{B}^{\prime} \rightarrow P_{B} \rightarrow X_{B} \rightarrow 0$ be an exact sequence with a projective $B$-module $P_{B}$. An application of $\operatorname{Hom}_{B}\left(-, Y_{B}\right)$ yields, for all $i \geq 0$, the exact sequences

$$
\operatorname{Ext}_{B}^{i}\left(P_{B}, Y_{B}\right) \rightarrow \operatorname{Ext}_{B}^{i}\left(X_{B}^{\prime}, Y_{B}\right) \rightarrow \operatorname{Ext}_{B}^{i+1}\left(X_{B}, Y_{B}\right) \rightarrow \operatorname{Ext}_{B}^{i+1}\left(P_{B}, Y_{B}\right) .
$$

Here, since $P_{B}$ is projective, the first and the last terms are zero for $i \geq 1$. Similarly, an application of $\operatorname{Hom}_{A}\left(-, Y_{A}\right)$ yields, for all $i \geq 0$, the exact sequences

$$
\operatorname{Ext}_{A}^{i}\left(P_{A}, Y_{A}\right) \rightarrow \operatorname{Ext}_{A}^{i}\left(X_{A}^{\prime}, Y_{A}\right) \rightarrow \operatorname{Ext}_{A}^{i+1}\left(X_{A}, Y_{A}\right) \rightarrow \operatorname{Ext}_{A}^{i+1}\left(P_{A}, Y_{A}\right) .
$$

Here, according to Statement 1, proj $\operatorname{dim} P_{A} \leq 1$, and thus the last term is zero for all $i \geq 1$. Moreover, $\operatorname{Ext}_{A}^{1}\left(P_{A}, Y_{A}\right) \simeq \operatorname{Ext}_{B}^{1}\left(P_{B}, Y_{B}\right)=0$, and therefore the first term is zero also for all $i \geq 1$. By induction, we may assume

$$
\operatorname{Ext}_{B}^{i}\left(X_{B}^{\prime}, Y_{B}\right) \simeq \operatorname{Ext}_{A}^{i}\left(X_{A}^{\prime}, Y_{A}\right)
$$

and

$$
\operatorname{Ext}_{B}^{i+1}\left(X_{B}, Y_{B}\right) \simeq \operatorname{Ext}_{A}^{i+1}\left(X_{A}, Y_{A}\right)
$$

as required.

StATEMENT 4. Let $J^{2}=J$ and $J_{A}$ be projective. Then

$$
\text { gl } \operatorname{dim} B \leq \mathrm{gl} \operatorname{dim} A \text {. }
$$

Proof. Let $g l \operatorname{dim} A=d<\infty$. If $X, Y$ are $B$-modules, then, in view of Statement 3, $\operatorname{Ext}_{B}^{d+1}\left(X_{B}, Y_{B}\right) \simeq \operatorname{Ext}_{A}^{d+1}\left(X_{A}, Y_{A}\right)=0$. Hence, gldim $B \leq d$.

StATEMENT 5. Let $A$ be a semiprimary ring with radical $N$. Let $J N J=0$ and $J_{A}$ be projective. Then

$$
\mathrm{gl} \operatorname{dim} A \leq \mathrm{gl} \operatorname{dim} B+2 .
$$


Proof. Let $\operatorname{gl} \operatorname{dim} B=d<\infty$. Given an $A$-module $X$, first calculate the projective dimension of the $A$-module $X J$. Let $\pi: P \rightarrow X J$ be a minimal projective cover. For every $x \in X$, let $J_{x}=J_{A}$, and let $P^{\prime}=\oplus_{x \in X} J_{x}$; finally, define $\pi^{\prime}: P^{\prime} \rightarrow X J$ by sending $y \in J_{x}$ to $x y$. Since $P^{\prime}$ is projective and $\pi^{\prime}$ is surjective, it follows that $P$ can be identified with a direct summand of $P^{\prime}$, and thus

$$
\text { ker } \pi \subseteq \operatorname{rad} P=P N \subseteq P^{\prime} N=\underset{x \in X}{\bigoplus} J_{x} N .
$$

Consequently, $(\operatorname{ker} \pi) J=0$, and thus $\operatorname{ker} \pi$ is a $B$-module. Therefore, by Statement 1, proj dim $(\operatorname{ker} \pi)_{A} \leq d+1$. Hence, proj dim $(X J)_{A} \leq d+2$.

On the other hand, $X / X J$ is a $B$-module, and thus, making use of Statement 1 again, proj $\operatorname{dim}(X / X J)_{A} \leq d+1$. Now, since $X$ is an extension of the $A$-modules $X J$ by $X / X J$ both of projective dimension $\leq d+2$,

$$
\text { proj } \operatorname{dim} X_{A} \leq d+2 \text {. }
$$

Statement 6. If $e$ is an idempotent of $A$, then $(A e A)^{2}=A e A$. Conversely, if $A$ is semiprimary and $J$ is an ideal of $A$ such that $J^{2}=J$, then $J=A e A$ for an idempotent $e$ of $A$.

Proof. The first assertion is trivial. So assume that $A$ is semiprimary with radical $N$ and that $J^{2}=J$. Any ideal of $A / N$ is generated by an idempotent, and any idempotent of $A / N$ is of the form $\bar{e}=e+N$ with an idempotent $e$ in $A$. Thus $J+N=A e A+N$ for some idempotent $e$ of $A$. Now, $J^{2}=J$ implies $(J+N)^{i}=J+N^{i}$ for all $i \geq 1$; similarly, $(A e A+N)^{i}=A e A+N^{i}$ for all $i \geq 1$. But for large $i, N^{i}=0$, and therefore $J=A e A$.

Statement 7. Let $e$ be an idempotent of a ring $A$. If the right module $(A e A)_{A}$ or the left module ${ }_{A}(A e A)$ is projective, then the multiplication map

$$
\mu: A e \underset{e A e}{\bigoplus_{e A}} e A \text { AeA }
$$

is bijective. Conversely, assume that $A$ is semiprimary with radical $N$ and that $e N e=0$. Then, if $\mu$ is bijective, both modules $(A e A)_{A}$ and ${ }_{A}(A e A)$ are projective.

Proof. For any $A$-module $X_{A}$, consider the multiplication map

$$
\mu_{X}: X \bigotimes_{A} A e \bigotimes_{e A e} e A \rightarrow X .
$$


The map $\mu_{X}$ is bijective for $X_{A}=e A$, and therefore for all direct summands of direct sums of the module $e A$. Now, if $(A e A)_{A}$ is projective, there is a surjective map of the form $\oplus e A \rightarrow A e A$, where the direct sum is indexed by all elements of $A$. Since this epimorphism splits, it follows that $\mu_{A e A}$ is bijective. But this means that $\mu$ is bijective. The same argument applies in the case that ${ }_{A}(A e A)$ is projective.

Now, assume that $A$ is semiprimary with radical $N$ and that $e N e=0$. Since $e N e=0$, the ring $e A e$ is simple artinian, and thus any $e A e$-module is projective. Since $(A e)_{e A e}$ and $(e A)_{A}$ are projective, $\left(A e \otimes_{e A e} e A\right)_{A}$ is projective also. Thus, the bijectivity of $\mu$ implies that $(A e A)_{A}$ is projective. Similarly, it implies that ${ }_{A}(A e A)$ is projective.

STATEMENT 8. Let $A$ be a semiprimary ring with radical $N$ and $J N J=0$. Then $L(A) \leq 2 L(B)+1$.

Proof. Since $A_{A}$ is an extension of $J_{A}$ by $B_{A}$, we have

$$
L(A) \leq L\left(J_{A}\right)+L(B) .
$$

Moreover, $J_{A}$ is an extension of $(J N)_{A}$ by $(J / J N)_{A}$. Since $J N J=0, J N$ is a $B$-module and thus $L\left((J N)_{A}\right) \leq L(B)$. On the other hand, $J / J N$ is semisimple and thus $L\left((J / J N)_{A}\right) \leq 1$. Consequently,

$$
L\left(J_{A}\right) \leq L\left((J N)_{A}\right)+L\left((J / J N)_{A}\right) \leq L(B)+1 .
$$

Now, making a subsequent use of Statements 7, 5 and 8, one can easily derive the following statement for quasi-hereditary rings.

STATEMENT 9. Let $A$ be a semiprimary quasi-hereditary ring with a heredity chain

$$
0=J_{0} \subseteq J_{1} \subseteq \cdots \subseteq J_{t} \subseteq \cdots \subseteq J_{m}=A .
$$

Then $A^{\mathrm{op}}$ is quasi-hereditary also, and

$$
0=J_{0}^{\mathrm{op}} \subseteq J_{1}^{\mathrm{op}} \subseteq \cdots \subseteq J_{t}^{\mathrm{op}} \subseteq \cdots \subseteq J_{m}^{\mathrm{op}}=A^{\mathrm{op}}
$$

is a heredity chain.

Moreover, $\operatorname{gldim} A \leq 2 m-2$ and $L(A) \leq 2^{m}-1$.

The following assertion can also be easily obtained.

STATEMENT 10. Let A be a semiprimary quasi-hereditary ring with a heredity chain

$$
0=J_{0} \subseteq J_{1} \subseteq \cdots \subseteq J_{t} \subseteq \cdots \subseteq J_{m}=A .
$$


Then, for each $1 \leq t \leq m, J_{t}=A e_{t} A$ for an idempotent $e_{t}$ of $A$ and

$$
0=e_{t} J_{0} e_{t} \subseteq e_{t} J_{1} e_{t} \subseteq \cdots \subseteq e_{t} J_{t} e_{t}=e_{t} A e_{t}
$$

is a heredity chain of $e_{t} A e_{t}$.

Proof. First, $J_{t}=A e_{t} A$ for some $e_{t}, e_{t}^{2}=e_{t}$, by Statement 6. Clearly,

$$
e_{t} J_{t} e_{t}=e_{t} A e_{t} A e_{t} \supseteq e_{t} A e_{t},
$$

and thus $e_{t} J_{t} e_{t}=e_{t} A e_{t}$. Now, if we show that $e_{t} J_{1} e_{t}$ is a heredity ideal of $e_{t} A e_{t}$, then the statement follows easily by induction. Since $J_{1}=A e_{1} A$ for an idempotent $e_{1}$ and $e_{1} \in A e_{t} A$,

$$
e_{t} J_{1} e_{t} \supseteq\left(e_{t} J_{1} e_{t}\right)^{2}=e_{t} A e_{1} A e_{t} A e_{1} A e_{t} \supseteq e_{t} A e_{1} A e_{t}=e_{t} J_{1} e_{t} .
$$

Furthermore, if $N$ is the radical of $A$, then $e_{t} N e_{t}$ is the radical of $e_{t} A e_{t}$ and

$$
\left(e_{t} J_{1} e_{t}\right)\left(e_{t} N e_{t}\right)\left(e_{t} J_{1} e_{t}\right) \subseteq e_{t} J_{1} N J_{1} e_{t}=0 .
$$

Finally, the $A$-module $\left(J_{1}\right)_{A}$ can be written as the finite direct sum of projective $A$-modules

$$
J_{1}=\bigoplus_{p} a_{p} A \oplus P
$$

where $P \subseteq\left(1-e_{t}\right) A, a_{p} A \simeq e A$ with $e^{2}=1, e e_{1}=e_{1} e=e$ and $a_{p}=e_{t} a_{p}=$ $a_{p} e_{1}$, i.e.,

$$
a_{p} \in e_{t} A e_{1} \subseteq e_{t} A e_{t} .
$$

Hence,

$$
e_{t} J_{1} e_{t}=\bigoplus_{p} a_{p}\left(e_{t} A e_{t}\right)
$$

is a decomposition into a direct sum of projective $e_{t} A e_{t}$-modules

$$
a_{p}\left(e_{t} A e_{t}\right) \simeq e A e_{t}=\left(e_{t} e e_{t}\right)\left(e_{t} A e_{t}\right) .
$$

Examples. Now we exhibit some examples (mostly path algebras over a field) to illustrate the necessity of some assumptions in the previous statements and the optimality of some bounds.

(i) The assumption that $J_{A}$ is projective cannot be omitted in Statements 1,2 and 5. 
Let $A$ be the path algebra of the diagram

$$
\circ \underset{\beta}{\stackrel{\alpha}{\rightleftarrows}} \text { 。 }
$$

modulo the ideal generated by $\alpha \beta$ and $\beta \alpha$. Let $J$ be a one-dimensional ideal. Then, $\operatorname{Hom}_{A}\left(J_{A}, B_{A}\right)=0, J^{2}=0 \neq J, J N J=0$,

$$
\mathrm{gl} \operatorname{dim} A=\infty \text { and } \mathrm{gl} \operatorname{dim} B=1 \text {. }
$$

(ii) The assumption $J^{2}=J$ cannot be omitted in Statements 3 and 4 .

Let $A$ be the path algebra of $\circ \rightarrow \circ \rightarrow \circ$ and $J$ the unique one-dimensional ideal. Then $J_{A}$ is projective, $J^{2}=0 \neq J$,

$$
\mathrm{gl} \operatorname{dim} A=1 \text { and } \mathrm{gl} \operatorname{dim} B=2 .
$$

(iii) The assumption that $J_{A}$ is projective cannot be omitted in Statements 3 and 4.

Let $A$ be the (basic) Auslander algebra of the algebra in (ii), i.e., the endomorphism algebra of the direct sum of all six indecomposable modules over the algebra in (ii). Let $J$ be the set of all endomorphisms which factor through the largest indecomposable (projective and injective) module. Then $J$ is an idempotent ideal,

$$
\operatorname{gl} \operatorname{dim} A=2 \text { and } \operatorname{gl} \operatorname{dim} B=4 .
$$

(iv) The assumption $J N J=0$ cannot be omitted in Statement 5 .

Let $A$ be the path algebra of the diagram

$$
1 \stackrel{\alpha}{\longrightarrow} 2 \supset \beta
$$

modulo the ideal generated by $\beta^{2}$. Let $J$ be the (idempotent) ideal AeA, where $e$ is the idempotent corresponding to the vertex 2 . Then $J_{A}$ is projective, $\operatorname{gl} \operatorname{dim} A=\infty$ and $\operatorname{gl} \operatorname{dim} B=0$.

(v) The assumption $e N e=0$ cannot be omitted in Statement 7 .

Let $A$ be the path algebra of the diagram

$$
1 \stackrel{\alpha}{\longrightarrow} 2 \supset \beta
$$

modulo the ideal generated by $\alpha \beta$ and $\beta^{2}$. Let $e$ be the idempotent corresponding to the vertex 2 . Then the multiplication map

$$
\text { Ae } \underset{e A e}{\otimes} e A \rightarrow A e A
$$

is bijective, ${ }_{A}(A e A)$ is projective and $(A e A)_{A}$ is not projective.

(vi) The bounds on global dimensions in Statements 5 and 9 are best possible. 
Let $A$ be the path algebra of the diagram

$$
1 \underset{\beta_{1}}{\stackrel{\alpha_{1}}{\rightleftarrows}} 2 \underset{\beta_{2}}{\stackrel{\alpha_{2}}{\rightleftarrows}} 3 \cdots m-1 \underset{\beta_{m-1}}{\stackrel{\alpha_{m-1}}{\rightleftarrows}} m
$$

modulo the ideal generated by all $\alpha_{t} \alpha_{t+1}, \beta_{t+1} \beta_{t}, \beta_{t} \alpha_{t}-\alpha_{t+1} \beta_{t+1}$ for $1 \leq t \leq$ $m-2$ and $\beta_{m-1} \alpha_{m-1}$. Let $e_{t}$ be the idempotent corresponding to the vertex $t$, $1 \leq t \leq m$. Put $J_{t}=A\left(e_{m}+e_{m-1}+\cdots+e_{m-t+1}\right) A$. Then $0=J_{0} \subset J_{1}$ $\subset \cdots \subset J_{t} \subset \cdots \subset J_{m}=A$ is a heredity chain of the quasi-hereditary algebra $A$ and $\operatorname{gl} \operatorname{dim} A=2 m-2$. Let us remark that $L(A)=3$.

(vii) The bounds on Loewy lengths in Statements 8 and 9 are best possible. Consider the complete oriented graph (without loops) on $m$ vertices; denote the arrow from the vertex $r$ to the vertex $s$ by $\alpha_{r s}, 1 \leq r, s \leq m, r \neq s$. Let $A$ be the path algebra of this graph modulo the ideal $I$ generated by all products $\alpha_{t_{1} t_{2}} \alpha_{t_{2} t_{3}} \cdots \alpha_{t_{k} t_{k+1}}$, where $t_{1}=t_{k+1}$ and all $t_{i}<t_{1}$ for $2 \leq i \leq k$. Let $e_{t}$ be the idempotent corresponding to the vertex $t, 1 \leq t \leq m$. Let

$$
J_{t}=A\left(e_{m}+e_{m-1}+\cdots+e_{m-t+1}\right) A .
$$

Then

$$
0=J_{0} \subset J_{1} \subset \cdots \subset J_{t} \subset \cdots \subset J_{m}=A
$$

is a heredity chain of the quasi-hereditary algebra $A$. Consider the element

$$
a=\alpha_{t_{1} t_{2}} \alpha_{t_{2} t_{3}} \cdots \alpha_{t_{k} t_{k+1}} \cdots \alpha_{t_{t^{m}-2} t_{2} m_{-1}} \text { modulo } I,
$$

where $t_{k}=r+1$ such that $k=2^{r}(2 s-1)$. It is not difficult to see that $a$ is a non-zero element of the socle of $A$, and to deduce, by induction, that $L(A)=2^{m}-1$. Let us remark that $\operatorname{gl} \operatorname{dim} A=2$.

\section{REFERENCES}

1. E. Cline, B. PARShall and L. ScotT, Algebraic stratification in representation categories, J. Algebra, to appear.

2. B. PARShALL and L. SCOTT, Derived categories, quasi-hereditary algebras and algebraic groups, Proceedings of Ottawa-Moosonee Workshop in Algebra, Carleton Univ. Notes, no. 3, 1988.

3. V. DLAB and C.M. RiNGEL, Auslander algebras as quasi-hereditary algebras, J. London Math. Soc., to appear

4. D. HAPPEL and C.M. Ringel, Tilted algebras, Trans. Amer. Math. Soc., vol. 274 (1982), pp. 339-443.

\section{CARLETON UNIVERSITY}

OtTáwa, OnTario, Canada

UNIVERSITÄT BIELEFELD

BieLEFELD, WeST GERMANY 